\title{
The Isolation and Some Properties of Radiation-sensitive Mutants of Micrococcus radiodurans
}

\author{
By B. E. B. MOSELEY* \\ Molteno Institute, Downing Street, Cambridge
}

(Accepted for publication I7 May 1967)

\begin{abstract}
SUMMAR Y
Treatment of the radiation-resistant bacterium Micrococcus radiodurans with ultraviolet (u.v.) radiation and $N$-methyl- $N^{\prime}$-nitro- $N$-nitrosoguanidine resulted in the isolation of two mutants highly sensitive to u.v. radiation. They were also sensitive to ionizing radiation and to the action of $N$-methyl$N^{\prime}$-nitro- $N$-nitrosoguanidine. The concentrations of sulphydryl groups in bacteria of the wild type and the mutants were not significantly different. Although the mutants were more sensitive to mitomycin $\mathrm{C}$ than the wild type the resistance of the latter was low. It is suggested that the DNA repair mechanism in the wild type operates very efficiently for the removal of single strand damage but not for that which involves cross-linking.
\end{abstract}

\section{INTRODUCTION}

Micrococcus radiodurans, a pigmented non-sporing bacterium, is characterized by its extreme resistance to ionizing radiation (Anderson, Nordan, Cain, Parrish \& Duggan, 1956) and to ultraviolet (u.v.) radiation (Duggan, Anderson, Elliker \& Cain, I959). The bacterial DNA, considered to be the radiation-sensitive target in other, more sensitive, bacteria, is not unusual in $M$. radiodurans either in its base composition or its quantity per cell (Moseley \& Schein, I964) and it is not exceptionally resistant to u.v. radiation damage as measured by the amount of the lethal photoproduct, thymine-dimer, formed on irradiation (Setlow \& Duggan, I964). There is now evidence for the existence in $M$. radiodurans of an efficient enzymic mechanism for the repair of u.v. damage, which excises thymine-dimers from its DNA with such efficiency that the eventual death of the bacteria appears to be due to other causes such as damage to deoxycytidine and protein (Setlow \& Boling, 1965; Boling \& Setlow, 1966). The repair mechanism is of the dark-repair type, similar to that found in u.v.-resistant strains of Escherichia coli (Setlow \& Carrier, I964; Boyce \& Howard-Flanders, I964a) but apparently operating with a much greater efficiency, since $M$. radiodurans can survive much higher doses of u.v. radiation. For example, the dose of u.v. radiation required to inactivate $90 \%$ of the organisms in a culture of the u.v.-resistant strain of E. coli $\mathrm{K} I 2 \mathrm{AB} I \mathrm{I} 57$ is about $1000 \mathrm{ergs} / \mathrm{mm}^{2}$ (Boyce \& Howard-Flanders, $1964 a$ ) while $15,000 \mathrm{ergs} / \mathrm{mm}^{2}$ is needed to achieve the same effect with $M$. radiodurans. There is also evidence that the resistance of $M$. radiodurans to ionizing radiation, which unlike u.v. radiation does not form thymine-dimers in DNA, is due to an enzymic repair mechanism for DNA damage (Moseley \& Laser, 1965a; Dean, Feldschreiber \& Lett, 1966). This mechanism

\footnotetext{
* Present address: Department of General Microbiology, University of Edinburgh, College of Agriculture, West Mains Road, Edinburgh, 9.
} 
appears to be essentially the same as that which operates for the repair of u.v. damage (Moseley \& Laser, 1965 $b$ ). The comparison of events which occur in u.v.-sensitive and u.v.-resistant strains of $E$. coli after u.v. radiation has greatly contributed to an understanding of the repair processes occurring in the resistant strains. Attempts have therefore been made to isolate radiation-sensitive mutants of $M$. radiodurans in order to establish by comparison the mechanism which confers on the wild type the unique property of being the vegetative bacterium which is most resistant to radiation.

\section{METHODS}

Organisms. The strain of Micrococcus radiodurans used was originally isolated by Anderson et al. (I956) and has been propagated in this laboratory for about 9 years. The bacteria grow in tetrads and contain a carotenoid pigment which gives colonies a salmon-pink appearance. The pigment can be extracted from dried bacteria with warm methanol and has an absorption peak at $475 \mathrm{~m} \mu$.

Media. TGYA broth for growth contained Bacto-Tryptone (Difco), $5 \mathrm{~g}$.; glucose, I g.; yeast extract (Difco), 3 g.; aspartic acid, 2 g.; distilled water, I 1., adjusted to pH $7 \cdot 2$ with $\mathrm{NaOH}$. TGYA agar for colony counts and replica plating was made by solidifying this medium with I $5 \mathrm{~g}$. Bacto agar/litre.

A buffer solution was used for suspending organisms during irradiation. It contained: $\mathrm{KH}_{2} \mathrm{PO}_{4},{ }_{13} .6$ g.; $\mathrm{Na}_{2} \mathrm{SO}_{4}, 2$ g.; $\mathrm{MgSO}_{4} .7 \mathrm{H}_{2} \mathrm{O}, 0.2$ g.; $\mathrm{Ca}\left(\mathrm{NO}_{3}\right)_{2} .4 \mathrm{H}_{2} \mathrm{O}, 0.0 \mathrm{O}$ g.; $\mathrm{FeSO}_{4} \cdot 7 \mathrm{H}_{2} \mathrm{O}, 0.5 \mathrm{mg}$.; distilled water to $\mathrm{I} 1$, adjusted to $\mathrm{pH} 7.2$ with $\mathrm{KOH}$. The same buffer was used for washing the bacteria and for dilution of suspensions for colony counts.

Preparation of organisms for irradiation. Colonies from an agar plate were inoculated into $12 \mathrm{ml}$. TGYA broth in $\perp$-tubes and the tubes shaken at $30^{\circ}$ for $\mathrm{I} 8 \mathrm{hr}$. The organisms were centrifuged down, washed and resuspended in buffer solution at a concentration of about $10^{8}$ colony forming units $/ \mathrm{ml}$. Care was taken to break up clumps of organisms by homogenising the suspensions immediately before irradiation by using an MSE homogeniser (Cat. No. 7700).

U.v. irradiation was done by using a Hanovia germicidal lamp (Model 12 ). $5 \mathrm{ml}$. samples of washed bacterial suspension were irradiated in Petri dishes $(9 \mathrm{~cm}$. diam.) at a distance of $40 \mathrm{~cm}$. from the lamp, the dose rate being $22.5 \mathrm{ergs} / \mathrm{mm} .{ }^{2} / \mathrm{sec}$. The suspension was agitated during irradiation by means of a magnetic stirrer to prevent sedimentation of the bacteria and to maintain uniformity of the absorbed dose.

$\gamma$-irradiation was carried out in a ${ }^{60} \mathrm{Co}$ source at a dose rate of $16.7 \mathrm{Krad} / \mathrm{min}$. Three ml. volumes of bacterial suspension were irradiated, oxygen being bubbled during the irradiation.

Isolation of mutants sensitive to u.v. radiation. Optimum conditions for the isolation of bacterial mutants usually result in the diminution of the colony count to about $0 \cdot \mathrm{I}-\mathrm{I} \cdot 0 \%$ of the initial count by treatment with the mutagen. However, $N$-methyl- $N^{\prime}$ nitro- $N$-nitrosoguanidine(NG) has been found to give high mutation rates in Escherichia coli at concentrations which caused only a 50\% loss of viability (Adelberg, Mandel \& Chen, I965). In contrast, very much higher concentrations of NG (500 $\mu \mathrm{g}$. $/ \mathrm{ml}$.) did not produce any loss of viability in Micrococcus radiodurans even after incubation for $3 \mathrm{hr}$. In view of the additive effect of ionising and u.v. damage (Moseley \& Laser, $1965 b$ ) it was decided to combine u.v. radiation with application of the mutagen. This was done 
by irradiating the bacteria with a non-lethal dose of u.v. radiation before exposing them to the mutagen. This combined treatment produced the desired decrease in viability with optimal chances of producing sensitive mutants.

Four $\mathrm{ml}$. of a suspension (about $\mathrm{IO}^{8}$ colony forming units $/ \mathrm{ml}$.) irradiated with I0,000 ergs $/ \mathrm{mm}^{2}$ of u.v. radiation were added to $5 \mathrm{ml}$. TGYA broth and the suspension shaken at $30^{\circ}$ for $30 \mathrm{~min}$. One $\mathrm{ml}$. NG solution $(5 \mathrm{mg} . / \mathrm{ml}$.) was added to a concentration of $500 \mu \mathrm{g}$. $/ \mathrm{ml}$. After $45 \mathrm{~min}$. further incubation the colony count was decreased to $0 \cdot 1-1 \cdot 0 \%$ of the original count. Samples ( $0 \cdot 1 \mathrm{ml}$.) were diluted Ioo-fold in TGYA broth, to dilute out the mutagen, and shaken at $30^{\circ}$ to allow one or two divisions to take place. Agar plates were then spread with dilutions of culture to give about $80-100$ colonies/plate and incubated at $37^{\circ}$ for 2 days. Two replicate copies of each plate were made by the felt-pad technique (Lederberg \& Lederberg, 1952). One copy was irradiated under the u.v. lamp for $20 \mathrm{~min}$. (a dose determined by sterilising small colonies of u.v.-sensitive Salmonella typhimurium CLT 22). After incubation for $24 \mathrm{hr}$. at $37^{\circ}$ the non-irradiated and irradiated plates were compared for growth. Colonies on the non-irradiated plates which were absent from the irradiated plates were isolated and screened for u.v. sensitivity.

Resistance of Micrococcus radiodurans to the lethal action of $N G$ and to mitomycin $C$. A sample $(2.5 \mathrm{ml}$.) of an $\mathrm{I} 8$-hr culture of $M$. radiodurans was added to $7.5 \mathrm{ml}$. TGYA broth and shaken at $30^{\circ}$ for $100 \mathrm{~min}$. (about I generation time). NG or mitomycin C was added to $100 \mu \mathrm{g} . / \mathrm{ml}$. and $20 \mu \mathrm{g}$. $/ \mathrm{ml}$., respectively, and $0 . \mathrm{I} \mathrm{ml}$. samples removed at suitable time intervals, diluted in buffer, and colony counts made on TGYA agar plates.

Determination of sulphydryl-group concentration in Micrococcus radiodurans. The technique was based on that of Hamm \& Hofmann (I965). To I ml. of a thick suspension of organisms (equiv. 50-70 mg. dry wt), $35 \mathrm{ml}$. of 8 M-urea and a slight excess of $\mathrm{AgNO}_{3}(3.5 \mu$ mole) were added. After stirring at room temperature for $\mathrm{I}$ hr to allow complete reaction between the $\mathrm{SH}$-groups and $\mathrm{AgNO}_{3}, 4.5 \mu$ mole of glutathione was added to give a slight excess of SH-groups. The excess of glutathione (as $\mathrm{SH}$ ) was then titrated amperometrically with $10^{-3} \mathrm{M}-\mathrm{AgNO}_{3}$.

\section{RESULTS}

\section{Isolation of mutants}

Two mutants of wild-type Micrococcus radiodurans which were sensitive to u.v. radiation were isolated. The two mutants are referred to as $M$. radiodurans UV 17 and $M$. radiodurans UV 38 . It was necessary before studying the new strains to show that they were mutants of $M$. radiodurans and not contaminants. The colonies of all three strains, wild type, UV 17 and UV 38 were similar in size and appearance, and all contained the carotenoid pigment which shows an absorption peak at $475 \mathrm{~m} \mu$. The concentration of pigment/unit dry wt organism was not significantly different in the three strains. Morphologically the organisms were identical and had the same mode of division which led to the formation of tetrads. Comparison of turbidity measurements with colony counts over the growth cycle gave similar plots, indicating that the dimensions of the organisms were similar.

Growth rates in TGYA broth were measured by using turbidity and colony count methods. In log-phase growth at $30^{\circ}$ with adequate aeration the generation time for 
the wild type was 90 min.; UV I 7 had the same generation time, but that of UV 38 was I 50 min., i.e. about $1 \cdot 7$ times longer. During the study of mutant UV I 7 another mutant arose spontaneously which was non-pigmented but which had all the other properties of UV 17, e.g. identical cell morphology, growth rate, and radiation sensitivity. This strain has been called $M$. radiodurans UV I $7 \mathrm{~W}$.

\section{Radiation resistance}

The dose response curves of the two mutants to u.v. radiation, compared with that of the wild type, are plotted in Fig. I which shows an enormous increase in sensitivity. The dose response curves to $\gamma$-radiation (Fig. 2) shows that even though the mutants were isolated on the basis of their u.v. sensitivity they were also sensitive to ionizing

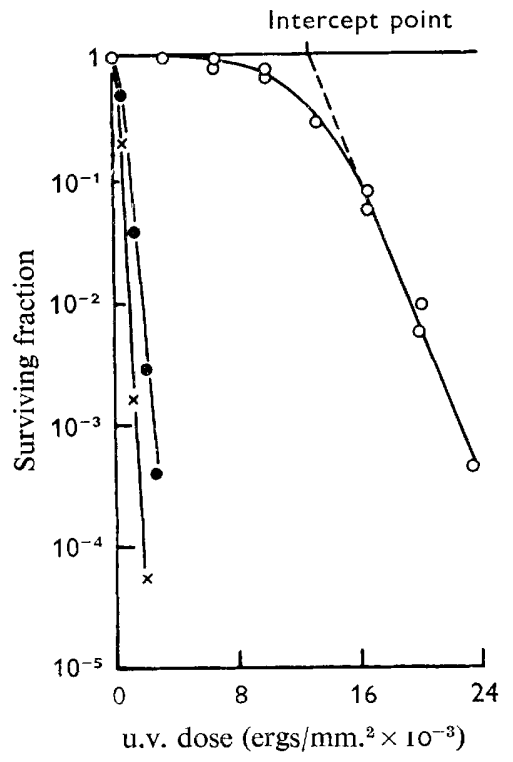

Fig. I

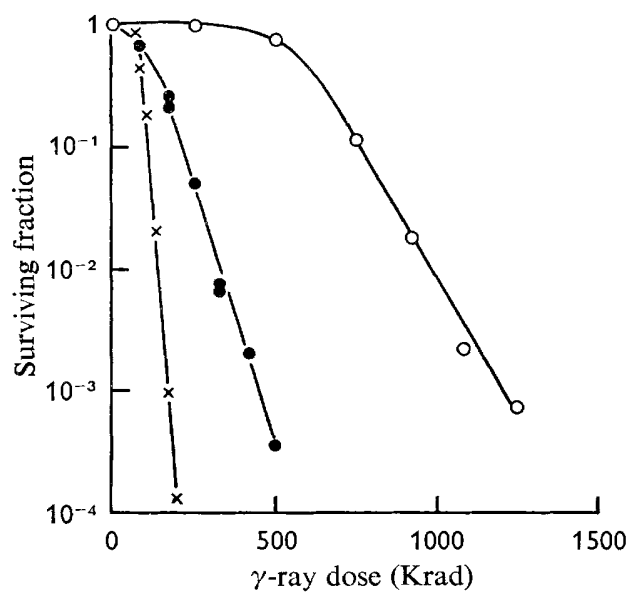

Fig. 2

Fig. I. Ultraviolet (u.v.) irradiation survival curves of Micrococcus radiodurans $(O)$ and the mutants UV $17(\times)$ and UV $38(-)$. The exponential portion of the survival curve of $M$. radiodurans has been extrapolated to unit survival to indicate the derivation of the intercept value used in Table $\mathrm{I}$.

Fig. 2. $\gamma$-irradiation survival curves of $M$. radiodurans $(O)$, UV $I 7(\times)$ and UV $38(\odot)$.

radiation. All the survival curves were sigmoidal. The shapes of the dose response curves have been defined by their intercept number, which is the dose obtained by extrapolating the exponential part of the curve to unit survival (see Fig. I). A summary of the results is given in Table $\mathrm{I}$.

Although the mutants were much more sensitive than the wild type, the increase in sensitivity to both types of radiation was not the same. A direct comparison of the survival curves is complicated by the fact that, with one exception, the increase in the exponential slope was not proportional to the reduction in the shoulder. Thus the reduction in the length of shoulder of strain UV 38 was much greater (28-fold for u.v. and five-fold for $\gamma$-radiation) than the increase in exponential slope, viz. five-fold for u.v.-radiation and two-fold for $\gamma$-radiation. Nevertheless, the data show that for 
Table I. Analysis of the dose-response curves shown in Figs. $I$ and 2

The $\mathrm{D}$ io value is the dose required to decrease the colony count of a culture by $90 \%$ on the exponential part of the survival curve. The intercept value, in terms of dose, is obtained by extrapolating the exponential part of the curve to unit survival. The ratios are derived from the $\mathrm{D}$ Io and intercept values of the various strains divided by those of the wild type.

\begin{tabular}{|c|c|c|c|c|c|c|c|c|}
\hline \multirow[b]{2}{*}{ Strain } & & &  & , & \multicolumn{4}{|c|}{$\gamma$-radiation dose response curves } \\
\hline & $\begin{array}{c}\text { D Io } \\
\text { (ergs/ } \\
\text { mm. }{ }^{2} \text { ) }\end{array}$ & Ratio & $\begin{array}{l}\text { cept } \\
\text { (ergs/ } \\
\left.\mathrm{mm}^{2}{ }^{2}\right)\end{array}$ & Ratio & $\begin{array}{l}D_{10} \\
(\mathrm{Kr})\end{array}$ & Ratio & $\begin{array}{l}\text { Inter- } \\
\text { cept } \\
(\mathrm{Kr})\end{array}$ & Ratio \\
\hline Wild type & 3,400 & I.O & 12,800 & $\mathrm{I} \cdot \mathrm{O}$ & 210 & $I \cdot O$ & 525 & $I \cdot O$ \\
\hline UV I 7 & 340 & $0 \cdot I$ & 450 & 0.035 & 30 & $0 \cdot 14$ & 75 & 0.14 \\
\hline UV 38 & 680 & 0.2 & 450 & 0.035 & 105 & 0.5 & 100 & 0.2 \\
\hline
\end{tabular}

strain UV 38 the increase in sensitivity to u.v. radiation was greater than that for $\gamma$-radiation. The same is true for strain UV 17 , the reduction in the shoulder being 28 -fold for u.v. radiation and 7 -fold for $\gamma$-radiation, while the exponential slope increased by factors of 10 and 7 respectively.

The lethal effect of $N$-methyl-N'-nitro-N-nitrosoguanidine ( $N G)$

Wild type Micrococcus radiodurans was completely resistant to the effect of $100 \mu \mathrm{g}$. $\mathrm{NG} / \mathrm{ml}$, , showing no loss of viability after $80 \mathrm{~min}$. of incubation. The radiation-sensitive mutants UV 17 and UV 38 were sensitive to the lethal action of NG, less than $\mathrm{IO}^{-3}$ of their populations surviving after 10 and $\mathrm{I} 5 \mathrm{~min}$. of incubation, respectively (Fig. 3). From this it follows that the increase in sensitivity of the mutants to NG was greater than that towards radiation. It was noted that practically all the colonies derived from wild-type organisms which had been incubated in the presence of NG I $00 \mu \mathrm{g} . / \mathrm{ml}$. for $80 \mathrm{~min}$. showed abnormalities, e.g. sectoring caused by partial loss of pigment, roughness, growth-rate variations, leading to the formation of very irregular edges to the colonies. On the other hand, colonies derived from surviving organisms of strains UV 17 and UV 38 were normal as compared with colonies from untreated organisms.

\section{The lethal effect of mitomycin $C$}

Figure 4 shows the lethal effect of mitomycin $\mathrm{C}$ at a concentration of $20 \mu \mathrm{g} . / \mathrm{ml}$. on Micrococcus radiodurans and its mutants UV $\mathrm{I} 7$ and UV 38 . The radiation-sensitive mutants were more sensitive to the action of mitomycin $\mathrm{C}$ than the wild type but the disparity was less than that for the lethal effect of u.v. radiation.

\section{Sulphydryl-group content}

Three samples of each bacterial strain were analysed for sulphydryl group content. Values obtained were $2 \mathrm{I}, 24$, and $27 \mu$ mole SH/g. dry wt of wild type, strain UV I 7 and strain UV 38 , respectively. The dry weight of a colony-forming unit of Micrococcus radiodurans obtained previously was $2.5 \times 10^{-12} \mathrm{~g}$. (Moseley \& Laser, 1965a) which gives values of $0.5,0.6$, and $0.7 \times 10^{-16}$ mole $\mathrm{SH} /$ colony-forming unit for the three strains. 


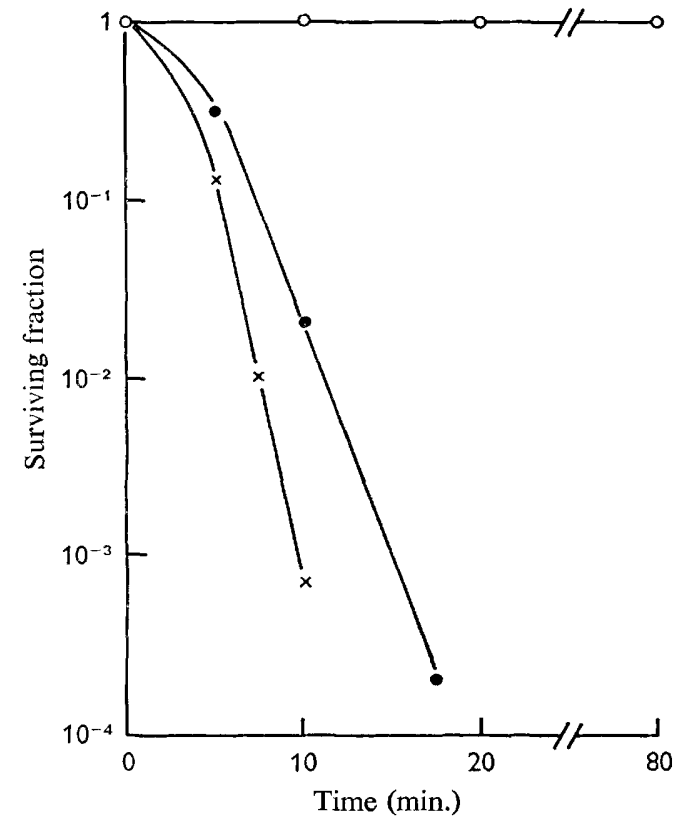

Fig. 3

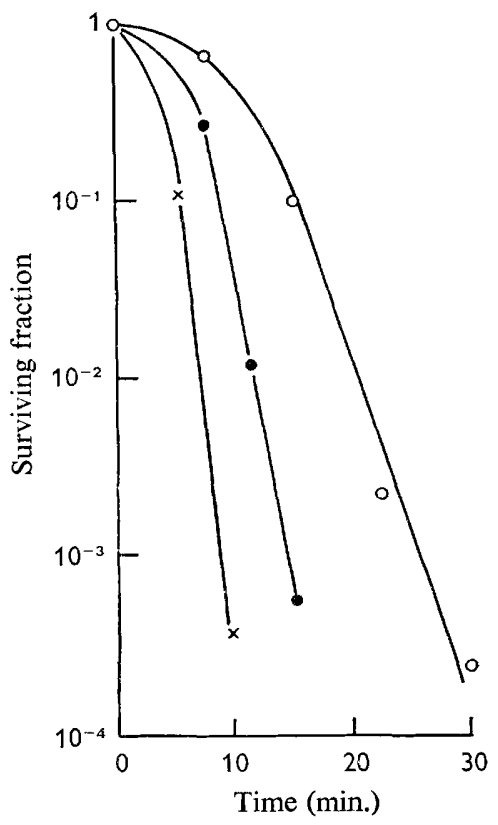

Fig. 4

Fig. 3. Resistance of $M$. radiodurans $(0)$, UV $\mathrm{I} 7(\times)$ and UV $38(\bullet)$ to incubation in NG $100 \mu \mathrm{g} . / \mathrm{ml}$.

Fig. 4. Resistance of $M$. radiodurans (O), UV $I 7(\times)$ and UV $38(\bullet)$ to incubation in mitomycin C $20 \mu \mathrm{g} . / \mathrm{ml}$.

\section{DISCUSSION}

The fact that Micrococcus radiodurans is the most resistant vegetative bacterium, so far investigated, to u.v. radiation as well as ionizing radiation has often been overlooked, and explanations have been sought for its resistance to ionizing radiation which have no relevance to its resistance to u.v. radiation. Some explanations have postulated that an intracellular carotenoid pigment (Kilburn, Bellamy \& Terni, I958) or a sulphydryl compound (Bruce, I964; Bruce \& Malchman, 1965) act as protectors. The isolation of non-pigmented mutants of $M$. radiodurans which have the same resistance as the wild type of ionizing radiation indicated that the pigment is not responsible for such resistance (Moseley \& Laser, 1965a) but it could not be excluded that pigment precursors were present which acted as energy-transfer substances. This possibility has now been excluded by the isolation of pigmented sensitive mutants.

The value of $0.5-0.6 \times \mathrm{IO}^{-16} \mathrm{~mole} \mathrm{SH} /$ colony-forming unit is in reasonable agreement with that obtained by Bruce \& Malchman (1965) of $0.8-2.0 \times 10^{-16}$ mole/organism based on the binding of $p$-hydroxymercuribenzoate. However, this concentration of sulphydryl groups is present in the radiation-sensitive organisms as well as in the radiation-resistant organisms. The mere presence of sulphydryl groups is not an indication of the presence of an intracellular protective compound able to confer such high resistance as is shown by the wild-type Micrococcus radiodurans. However, some of the residual resistance to ionizing radiation in the sensitive strains may yet be due to the presence of sulphydryl groups and thus account for the fact that they 
are slightly less sensitive to ionizing radiation than to u.v. radiaton. This is a very small part of the total resistance of the wild-type $M$. radiodurans to ionizing radiation, the major part of which is due to the presence of a repair mechanism.

The dark repair system which operates in u.v. resistant strains of Escherichia coli does not only recognize damage to DNA of the thymine-dimer type. The excision of defective bases and the incorporation of new ones follows treatment of organisms with the bifunctional alkylating agent nitrogen mustard (Hanawalt \& Haynes, 1965) and with NG (Hanawalt, I966). Thus the precise base defect appears to be less important than some associated secondary structural alteration in the phosphodiester backbone of the DNA. This situation is also true for Micrococcus radiodurans. Its remarkable capacity to survive extremely high doses of ionizing radiation and of u.v. radiation is complemented by its resistance to the decay of radioactive phosphorus $\left({ }^{32} \mathrm{P}\right)$ incorporated in its DNA and to the action of NG, which probably acts as a monofunctional alkylating agent. Micrococcus radiodurans organisms remain viable even after 50,000 ${ }^{32} \mathrm{P}$ disintegrations/nucleus, as compared with values of $10-50$ for $E$. coli (M. Swann, personal communication). Since $M$. radiodurans has such great resistance to radiation and to compounds which cause DNA defects, it is surprising that it is sensitive to mitomycin C which cross-links DNA in vivo (lyer \& Szybalski, I963) and which functions partially as a bifunctional alkylating agent (Iyer \& Szybalski, 1964). Although wildtype $M$. radiodurans is more resistant to mitomycin $C$ than are the radiation-sensitive mutants, the order of resistance is quite low, being the same as that shown by u.v.resistant strains of $E$. coli (Boyce \& Howard-Flanders, I964b). This suggests that $M$. radiodurans has a repair system of extremely high efficiency for damage within one or both strands of the DNA, but which does not operate for damage involving crosslinkage. The higher resistance of wild-type $M$. radiodurans as compared with that of the sensitive mutants need not be interpreted in terms of removal of cross-links since only I out of 5 to Io mitomycin C molecules participates in the formation of crosslinks while the others react with one DNA strand only (Iyer \& Szybalski, 1964; Weissbach \& Lisio, 1965). This suggests that the differential sensitivity of the three strains of $M$. radiodurans to mitomycin $\mathrm{C}$ reflects the inability of the radiation-sensitive strains to repair monofunctional alkylating damage which the wild type repairs very efficiently (hence its high resistance to $\mathrm{NG}$ ) but is inactivated by cross-link damage. Preliminary biochemical studies indicate that the radiation-sensitive mutants and wild-type $M$. radiodurans are able to excise u.v. radiation-induced thymine dimers from their DNA and are able to incorporate fresh bases. Their sensitivity appears to be due to the inability to control the excision process (Moseley, 1967).

I wish to thank Dr G. W. Gould for the sulphydryl analysis and Dr. H. Laser for valuable discussions.

This work was supported by a grant from the Medical Research Council. 


\section{REFERENCES}

Adelberg, E. A., Mandel, M. \& Chen, G. C. C. (1965). Optimal conditions for mutagenesis by $N$-methyl- $N^{\prime}$-nitro- $N$-nitrosoguanidine in Escherichia coli $\mathrm{K} 12$. Biochem. biophys. Res. Commun. I8, 788 .

Anderson, A. W., Nordan, H. C., Cain, R. F., Parrish, G. \& Duggan, D. (1956). Studies on a radioresistant micrococcus. I. Isolation, morphology, cultural characteristics and resistance to gamma radiation. Fd Technol., Champaigne. Io, 575.

Boling, M. E. \& Setlow, J. K. (1966). The resistance of Micrococcus radiodurans to ultraviolet radiation. III. A repair mechanism. Biochim. Biophys. Acta I23, 26.

Boyce, R. P. \& Howard-Flanders, P. (I964a). Release of ultraviolet light-induced thymine dimers from DNA in E. coli K-I2. Proc. natn. Acad. Sci., U.S.A. 5I, 293.

Boyce, R. P. \& Howard-Flanders, P. (1964b). Genetic control of DNA breakdown and repair in E. coli $\mathrm{K}-\mathrm{I} 2$ treated with mitomycin C or ultraviolet light. Z. VererbLehre $\mathbf{9 5}, 345$.

BrUCE, A. K. (1964). Extraction of the radioresistant factor of Micrococcus radiodurans. Radiat. Res. 22, 155 .

BRUCE, A. K. \& Malchman, W. H. (1965). Radiation sensitisation of Micrococcus radiodurans, Sarcina lutea, and Escherichia coli by p-hydroxymercuribenzoate. Radiat. Res. 24, 473.

Dean, C. T., Feldschreiber, P. \& Lett, J. T. (I966). Repair of X-ray damage to the deoxyribonucleic acid in Micrococcus radiodurans. Nature, Lond. 209, 49.

Duggan, D. E., Anderson, A. W., Elliker, P. R. \& Cain, R. F. (1959). Ultraviolet exposure studies on a gamma radiation resistant micrococcus isolated from food. Fd Res. 24, 376.

HAMm, R. \& HOFMANN, K. (1965). Changes in the sulphydryl and disulphide groups in beef muscle proteins during heating. Nature, Lond. 207, 269.

HaNawalt, P. C. (I966). Repair replication in the bacterial genome. In: Genetical Aspects of Radiosensitivity: Mechanisms of Repair. Vienna: International Atomic Energy Agency.

Hanawalt, P. C. \& Haynes, R. H. (I965). Repair replication of DNA in bacteria: irrelevance of chemical nature of base defect. Biochim. Biophys. Res. Commun. I9, 462.

IYER, V. N. \& SZYBALSKI, W. (I963). A molecular mechanism of mitomycin action: linking of complementary DNA strands. Proc. natn. Acad. Sci., U.S.A. 50, 355.

IYER, V. N. \& SZYBALSKI, W. (I964). Mitomycins and porfiromycin: chemical mechanisms of activation and cross linking of DNA. Science $\mathbf{1 4 5}, 55$.

Kilburn, R. E., Bellamy, W. D. \& Terni, S. A. (1958). Studies on a radiation-resistant pigmented Sarcina sp. Radiat. Res. 9, 207.

Lederberg, J. \& LederberG, E. M. (1952). Replica plating and indirect selection of bacterial mutants. J. Bact. 63, 399.

Moseley, B. E. B. (1967). The repair of DNA in Micrococcus radiodurans following ultraviolet irradiation. J. gen. Microbiol. 48, vi.

Moseley, B. E. B. \& LASER, H. (I965a). Repair of X-ray damage in Micrococcus radiodurans. Proc. Roy. Soc. B I62, 2 IO.

Moseley, B. E. B. \& LASER, H. (I965 b). Similarity of repair of ionizing and ultraviolet radiation damage in Micrococcus radiodurans. Nature, Lond. $206,373$.

Moseley, B. E. B. \& SChein, A. H. (I964). Radiation resistance and deoxyribonucleic acid base composition of Micrococcus radiodurans. Nature, Lond. 203, I 298.

SEtlow, J. K. \& Boling, M. E. (1965). The resistance of Micrococcus radiodurans to ultraviolet radiation. II. Action spectra for killing, delay in DNA synthesis, and thymine dimerisation. Biochim. Biophys. Acta xo8, 259.

Setlow, J. K. \& Duggan, D. E. (1964). The resistance of Micrococcus radiodurans to ultraviolet radiation. I. Ultraviolet-induced lesions in the cell's DNA. Biochim. Biophys. Acta 87, 664.

SETLOW, R. B. \& CARRIER, W. L. (1964). The disappearance of thymine dimers from DNA: An error correcting mechanism. Proc. natn. Acad. Sci. U.S.A. 51, 226.

WeissBaCH, A. \& Lisio, A. (1965). Alkylation of nucleic acids by mitomycin C and porfiromycin. Biochemistry 4, 196. 